\title{
Scattering Theory of Photon-Assisted Electron Transport
}

\author{
Morten Holm Pedersen and Markus Büttiker \\ Département de Physique Théorique, Université de Genève, 1211 Genève 4, Switzerland
}

(April 8, 2022)

\begin{abstract}
The scattering matrix approach to phase-coherent transport is generalized to nonlinear actransport. In photon-assisted electron transport it is often only the dc-component of the current that is of experimental interest. But ac-currents at all frequencies exist independently of whether they are measured or not. We present a theory of photon-assisted electron transport which is charge and current conserving for all Fourier components of the current. We find that the photo-current can be considered as an up- and down-conversion of the harmonic potentials associated with the displacement currents. As an example explicit calculations are presented for a resonant double barrier coupled to two reservoirs and capacitively coupled to a gate. Two experimental situations are considered: in the first case the ac-field is applied via a gate, and in the second case one of the contact potentials is modulated. For the first case we show that the relative weight of the conduction sidebands varies with the screening properties of the system. In contrast to the non-interacting case the relative weights are not determined by Bessel functions. Moreover, interactions can give rise to an asymmetry between absorption and emission peaks. In the contact driven case, the theory predicts a zero-bias current proportional to the asymmetry of the double barrier. This is in contrast to the discussion of Tien and Gordon which, in violation of basic symmetry principles, predicts a zero-bias current also for a symmetric double barrier.
\end{abstract}

PACS numbers: 73.23.-b, 85.30.Vw, 73.40.Gk, 72.10.-d

\section{INTRODUCTION}

Photon-assisted tunneling has been of interest since the work of Tien and Gordon [1] and Tucker [2]. Carrier transmission through barriers with oscillating potentials has been analyzed to find the traversal time for tunneling [3]. Recently photon-assisted tunneling has found renewed interest in the field of mesoscopic physics stimulated by theoretical work by Bruder and Schoeller [4] and experiments on quantum dots by Kouwenhoven and McEuen et al. [5], and by experiments on superlattices by the group of Allen et al. [6] 8 . Typically of interest [4,5,7 24] is the zero-frequency current component induced in response to an oscillating voltage. Theoretical treatments of photon-assisted electron transport often assume that the driving field is known and equals the external field. However, the long-range Coulomb interaction will screen the external field and generates an internal potential that can be quite different from the applied potential. Similarly, since it is the dc-component which is measured, one might think that displacement currents play no role. However, the dc-component is a consequence of nonlinearities in the conduction process. Clearly, in such a conductor, the current has not only a dc-component, but also currents at the frequency of the oscillating voltage and its higher harmonics. Not only are the dc-currents conserved but also the currents at the oscillation frequency and at its higher harmonics. Consequently a theory is needed in which all frequency components of the current are treated self-consistently. Such a theory is developed below. It leads to the conclusion that the photo-current is induced by a non-linear upand down-conversion of the electric-fields (potentials) as- sociated with the displacement current. Bessel functions are often a hall mark [1] of the discussion of photonassisted tunneling: However, in the self-consistent theory discussed below Bessel functions cannot in general describe the relative weights of the sideband peaks since the argument of the Bessel functions is not invariant under an overall potential shift. Since in nonstationary conditions charge accumulation occurs and causes induced fields, a selfconsistent treatment of the electron-electron interactions is important. The issues are similar for theories and experiments which investigate photon-assisted process not in the dc-current but in its fluctuations [25,26]. Here we emphasize mainly the average current and address the fluctuation spectra only briefly in an appendix.

A convenient description of conduction processes in mesoscopic systems which incorporates the role of contacts and permits to investigate directly the phasecoherent transmission from one reservoir to another, is the scattering matrix approach [27,28. The description of linear ac-conduction in response to oscillating potentials and consideration of the long range Coulomb interaction has already been discussed both for the case of zero-dimensional systems [29,30] and for extended systems for which one needs to discuss the entire potential landscape [31,32]. A review of this subject can be found in Ref. 33]. Here we generalize the scattering matrix approach to take into account the nonlinear dependence on oscillating potentials. First we consider the response of the electrons to a potential applied only to the contacts of the sample, assuming the internal potential is kept fixed. The response to the total potential will, in a subsequent step, be calculated selfconsistently in randomphase-approximation (RPA). The resulting charge and 
current conserving theory will be used to investigate the photo-induced dc-current in a resonant tunneling barrier. As function of Fermi energy and frequency we find large differences between the induced internal potential and the external applied potential, showing that long range Coulomb forces are important for photon-assisted tunneling in mesoscopic systems. Furthermore, interactions can give rise to an asymmetry between absorption and emission peaks, as well as changing the distance between peaks from a multiple of photon quanta to a distance depending on screening properties.

Our discussion is complementary to works which model interactions based on a Hamiltonian suitable to describe Coulomb blockade effects. The work of Bruder and Schoeller th also considers coupling to a gate and also considers displacement currents. In principle, all the questions addressed here can be investigated within such a framework. The scattering approach used here has the advantage that it is not limited to the tunneling regime but can also be applied to conductors which are strongly coupled to reservoirs (ballistic or metallic diffusive wires, etc). The RPA treatment as it is formulated below does have the disadvantage that it is not an appropriate description in the case when charge quantization effects (Coulomb blockade) are important. However, its conceptual clarity makes the RPA treatment a useful point of reference for comparision of different theoretical discussions.

The basic view taken here is the same as that used for the discussion of dc-conductances 28] and acconductance [29]. What is needed is the connection between currents at the contacts of the structure and the voltages at these contacts. Either the currents or the voltages can be controlled. As in the discussion of the ac-conductance it is necessary to consider not only the mesoscopic conductor itself but all nearby metallic bodies (gates and capacitors) which interact via long range Coulomb forces with the mesoscopic conductor. Let $\alpha$ label all the relevant contacts. The current at contact $\alpha$ can be written in terms of its Fourier components $I_{\alpha}(n \omega)$. Here $n=0$ is the dc component of the currents, and $n= \pm 1$ are the Fourier components at the driving frequency. Nonlinearities lead to higher harmonics $n= \pm 2,3, \ldots$ Similarly, the voltage at contact $\alpha$ has the Fourier components $V_{\alpha}(n \omega)$. We emphasize that the voltage of a contact is only a well defined quantity if local electric fields deep inside the contact vanish. There must, therefore, exist a Gauss volume which encloses the mesoscopic conductor [29]. The electric flux through this Gauss volume vanishes. As a consequence the total charge $Q$ inside the volume is conserved 229]. Charge conservation, and current conservation, apply to each Fourier component separately. In particular, we must have that the total charge within the Gauss volume vanishes at each frequency,

$$
Q_{\alpha}(n \omega)=0 .
$$

A theory for which this holds gives currents which depend ultimately only on voltage differences. We call such a theory of electric conductance gauge invariant 34]. To be definite let us introduce an expansion parameter $\epsilon$. We take the Fourier components of the first harmonic $V_{\alpha}(\omega)$ proportional to $\epsilon$ and expand the currents in powers of $\epsilon$. The second harmonic voltages $V_{\alpha}(2 \omega)$ describing twophoton processes are then proportional to $\epsilon^{2}$. Below we write the relationship between currents and voltages up to second order in $\epsilon$. The expansion coefficients are conductances $g_{\alpha \beta \gamma}(n \omega, m \omega)$ which give the current at contact $\alpha$ in response to a voltage $V_{\beta}(n \omega)$ at contact $\beta$ at a frequency $n \omega$ and a voltage a contact $\gamma$ at a frequency $m \omega$. The overall dc-current is

$$
\begin{aligned}
I_{\alpha}(0) & =I_{\alpha}^{d c}\left[\left\{V_{\beta}(0)\right\}\right]+I_{\alpha}^{p h}\left[0 ;\left\{V_{\beta}(0)\right\}\right], \\
I_{\alpha}^{p h}\left[0 ;\left\{V_{\beta}(0)\right\}\right] & =\sum_{\beta \gamma} g_{\alpha \beta \gamma}\left[\omega,-\omega ;\left\{V_{\delta}(0)\right\}\right] V_{\beta}(\omega) V_{\gamma}^{*}(\omega) .
\end{aligned}
$$

The first term of Eq. (2) , $I_{\alpha}^{d c}\left[\left\{V_{\beta}(0)\right\}\right]$, is the direct current that would be measured in the presence of purely static voltages $V_{\beta}(0), \beta=1,2, .$. applied to the different contacts of the sample. In the following, for the direct current, we retain the full dependence to all orders in the static applied voltages. If the dc-current $I_{\alpha}^{d c}\left[\left\{V_{\beta}(0)\right\}\right]$ is expanded in powers of the applied voltage then the terms linear in the applied voltages determine the dcconductance matrix $g_{\alpha \beta}(0)$ and the terms quadratic in the applied voltages are the dc-rectification conductances $g_{\alpha \beta \gamma}(0)$, discussed by Christen and one of the authors [35,29, which determine the leading order non-linearity of the dc I-V-characteristic [36]. In addition to these contributions to the dc-current which characterize the purely stationary transport there is now also a contribution to the dc-current due to the photon-assisted processes, $I_{\alpha}^{p h}\left[0 ;\left\{V_{\beta}(0)\right\}\right]$. In particular, to second order in the applied ac-voltages $V_{\beta}(\omega)$, carriers which emit and re-absorb (virtual) photons are determined by the dcphoto-conductance $g_{\alpha \beta \gamma}\left[\omega,-\omega ;\left\{V_{\delta}(0)\right\}\right]$ which depends in general also on the dc-voltages $V_{\delta}(0)$. These conductance coefficients represent an up- and down-conversion of the first harmonic voltages.

The current at the frequency of the oscillating potential is in general composed both of a particle current and of a displacement current. To be brief we call this current simply the displacement current. To linear order in our expansion parameter it is given by

$$
I_{\alpha}(\omega)=\sum_{\beta} g_{\alpha \beta}\left[\omega ;\left\{V_{\gamma}(0)\right\}\right] V_{\beta}(\omega) .
$$

Here expanding $g_{\alpha \beta}\left[\omega ;\left\{V_{\gamma}(0)\right\}\right]$ in the dc voltages yields the equilibrium admittance [30] of the mesoscopic structure $g_{\alpha \beta}(\omega)$ and the dc-ac-rectification conductance $g_{\alpha \beta \gamma}(\omega ; 0)$.

The current at $2 \omega$ is

$$
I_{\alpha}(2 \omega)=\sum_{\beta} g_{\alpha \beta}\left[2 \omega ;\left\{V_{\gamma}(0)\right\}\right] V_{\beta}(2 \omega)
$$




$$
+\sum_{\beta \gamma} g_{\alpha \beta \gamma}\left[\omega, \omega ;\left\{V_{\delta}(0)\right\}\right] V_{\beta}(\omega) V_{\gamma}(\omega)
$$

determined by a second harmonic conductance $g_{\alpha \beta}\left[2 \omega ;\left\{V_{\gamma}(0)\right\}\right]$ and a non-linear up-conversion conductance $g_{\alpha \beta \gamma}\left[\omega, \omega ;\left\{V_{\delta}(0)\right\}\right]$ whereby a second harmonic current is generated due to a non-linear combination of first harmonic voltages. We emphasize that the expansion given here can in principle be carried further to an arbitrary order in $\epsilon$. Our task is to find explicit expressions for the (non-linear) ac-conductances defined in Eqs. (2 - 5). It is useful, to state first a number of general properties of these conductances.

Current conservation holds for each Fourier component separately. Furthermore, since we can break off the expansion at any order, current conservation restricts each type of conductance coefficient in Eqs. (2-5). An additional restriction imposed on these conductance coefficients arises due to the fact that a voltage $V(n \omega)$ which is applied to all contacts simultaneously can not have a physical effect. As a consequence the conductances obey the sum rules 35,29]

$$
\sum_{\alpha} g_{\alpha \beta}(k \omega)=\sum_{\beta} g_{\alpha \beta}(j \omega)=0
$$

for $k, j \in \mathcal{N}$. Similarly, the second order coefficients obey

$$
\begin{aligned}
\sum_{\alpha} g_{\alpha \beta \gamma}(k \omega, j \omega)= & \sum_{\beta} g_{\alpha \beta \gamma}(k \omega, j \omega) \\
& =\sum_{\gamma} g_{\alpha \beta \gamma}(k \omega, j \omega)=0 .
\end{aligned}
$$

These sum rules guarantee that the final result will depend on voltage differences only.

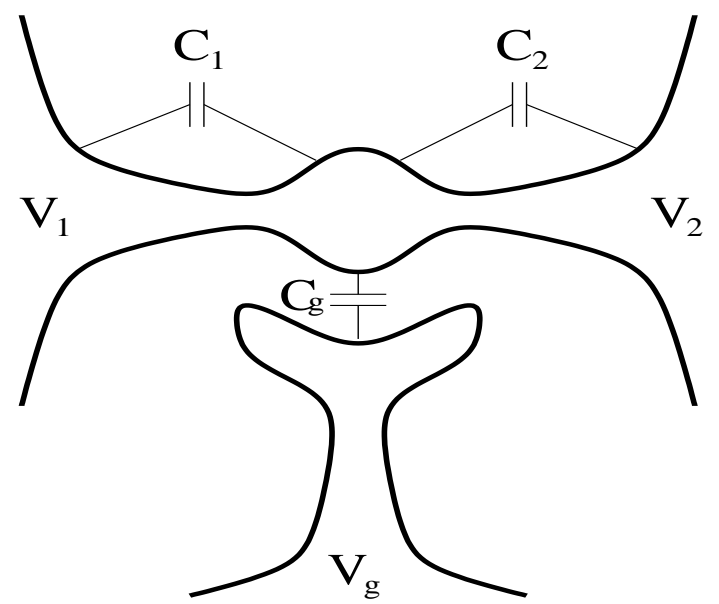

FIG. 1. Conductor connected to two contacts and coupled capactively to a gate.

Eqs. (2 - 5) are completely general and are applicable to any phase-coherent multi-terminal conductor. We now discuss these general relations for the case of a twoterminal conductor capacitively coupled to a gate, a situation sketched in Fig. 1, in the limit $C_{1}=C_{2}=0$.
This simple arrangement permits us already to point to the connection between photo-currents and displacement currents. We are interested in the photo-current generated by a sinusoidal oscillation of the voltage $V_{g}(\omega)$ at the gate. First consider the displacement current. The oscillating gate couples with the conductor in a purely capacitive manner. Therefore, the $g_{\alpha g}\left[\omega ;\left\{V_{\beta}(0)\right\}\right]$ describe capacitive currents and we can write $g_{\alpha g}\left[\omega ;\left\{V_{\beta}(0)\right\}\right]=$ $-i \omega C_{\alpha g}\left[\omega ;\left\{V_{\beta}(0)\right\}\right]$. We emphasize that this is a global transport coefficient which connects the voltage at one contact to the current at another contact. As a consequence, the capacitance coefficients are not of a purely geometrical nature but can be strong functions of magnetic field and the dc gate voltage [29, 37, 34, 38]. Thus, the current at contact $\alpha$ is determined by

$$
I_{\alpha}(\omega)=-i \omega C_{\alpha g}\left[\omega ;\left\{V_{\beta}(0)\right\}\right] V_{g}(\omega) .
$$

Next, consider the dc-photo-current generated by this arrangement

$$
I_{\alpha}^{p h}(0)=g_{\alpha g g}\left[\omega,-\omega ;\left\{V_{\beta}(0)\right\}\right]\left|V_{g}(\omega)\right|^{2} .
$$

With any of the equations for the displacement current we can eliminate the oscillating gate voltage entirely and find

$$
I_{\alpha}^{p h}(0)=\frac{1}{\omega^{2}} \frac{g_{\alpha g g}\left[\omega,-\omega ;\left\{V_{\beta}(0)\right\}\right]}{\left|C_{\alpha g}\left[\omega ;\left\{V_{\beta}(0)\right\}\right]\right|^{2}}\left|I_{\alpha}(\omega)\right|^{2} .
$$

Thus to second order in the oscillating voltages, the photo-current is directly related to the displacement current. Since the displacement current is not a property of a non-interacting system but is in an essential way determined by the long range Coulomb interaction, so similarly, the long range Coulomb interaction must play an essential role in determining the photo-current. Note that the photo-conductance which enters Eq. (10) is also proportional to $\omega^{2}$ and the photo-current given in Eq. (10) therefore has a well defined zero-frequency limit.

Now we proceed to find explicit expressions for the non-linear conductances introduced above.

\section{OSCILLATING CONTACT POTENTIALS: EXTERNAL RESPONSE}

We consider a conductor with voltages which oscillate in time applied to the contacts of the sample or to nearby capacitors. First we evaluate the response of non-interacting particles with the internal potential kept fixed. Only the response to the total potential has physical meaning, however, these results are needed in the next section for treating the problem with interactions.

The current operator for current incident in contact $\alpha$ in a mesoscopic system can be written as [39]

$$
\begin{aligned}
\hat{I}_{\alpha}(t)= & \frac{e}{h} \int d E \int d E^{\prime}\left[\hat{\mathbf{a}}_{\alpha}^{\dagger}(E) \hat{\mathbf{a}}_{\alpha}\left(E^{\prime}\right)\right. \\
& \left.-\hat{\mathbf{b}}_{\alpha}^{\dagger}(E) \hat{\mathbf{b}}_{\alpha}\left(E^{\prime}\right)\right] e^{i \frac{E-E^{\prime}}{h} t}
\end{aligned}
$$


where $\hat{\mathbf{a}}_{\alpha}$ and $\hat{\mathbf{b}}_{\alpha}$ are vectors of operators with components $\hat{a}_{\alpha n}$ and $\hat{b}_{\alpha n}$. Here $\hat{a}_{\alpha n}$ annihilates an incoming carrier in channel $n$ in lead $\alpha$ and $\hat{b}_{\alpha n}$ annihilates an outgoing carrier in channel $n$ in lead $\alpha$. Eq. (11) applies for frequencies $\left(E-E^{\prime}\right) / \hbar$ small compared to the Fermi energy.

The incoming and outgoing waves are related by the scattering matrix $39 \mathbf{s}_{\alpha \beta}$ via, $\hat{\mathbf{b}}_{\alpha}=\sum_{\beta} \mathbf{s}_{\alpha \beta} \hat{\mathbf{a}}_{\beta}$. In a multichannel conductor the s-matrix has dimensions $N_{\alpha} \times N_{\beta}$ for leads with $N_{\alpha}$ and $N_{\beta}$ channels. Here, and in the following, greek indices run over all contacts of the conductors.

Let us now suppose that a potential variation is applied to reservoir $\alpha$. The potential is $e U_{\alpha}(t)=e V_{\alpha}(\omega) \cos \omega t$, where $V_{\alpha}(\omega)$ is the modulation amplitude. With this potential the solution to the single-particle Schrödinger equation at energy $E$ in $\alpha$ is

$$
\psi_{\alpha, n}(x, t ; E)=\phi_{\alpha, n}(x ; E) e^{-i E t / \hbar} \sum_{l=-\infty}^{\infty} J_{l}\left(\frac{e V_{\alpha}}{\hbar \omega}\right) e^{-i l \omega t}
$$

where $\phi_{\alpha, n}(x ; E)$ is the wave function describing an incoming (or outgoing) carrier in contact $\alpha$ in channel $n$ in the absence of a modulation potential, and $J_{l}$ is the $l$ 'th order Bessel function. Thus the potential modulation leads for each state with central energy $E$ to side bands at energy $E+l \hbar \omega$ describing carriers which have absorbed $l>0$ modulation quanta or have emitted $l<0$ modulation quanta $\hbar \omega$. Here we have assumed that all potentials oscillate in phase. If one allows for a different phase $\phi_{\alpha}$ for each contact $\alpha$ that will add a term $e^{-i l \phi_{\alpha}}$ to each term in the sum in the wave-function above. Below, for simplicity, we assume that all contact potentials are in phase.

We now suppose that the modulation potential exists only far away from the conductor and that the modulation potential vanishes as we approach the conductor. Thus there is a transition region from a portion of the lead in which the potential is oscillating and a portion of the lead close to the conductor where we initially assume that the potential is time-independent and equal to the equilibrium potential. Now we need the wave function in the time-independent potential region. This leads to a matching problem. If the transition is adiabatic a state with energy $E$ in the conductor obtains a contribution from all reservoir states with central energy $E-l \hbar \omega$ due to its side band of amplitude $J_{l}\left(\frac{e V_{\alpha}}{\hbar \omega}\right)$ at energy $E$. In the notation of second quantization the annihilation operator of an incoming state close to the conductor is

$$
\hat{\mathbf{a}}_{\alpha, n}(E)=\sum_{l} \hat{\mathbf{a}}_{\alpha, n}^{\prime}(E-l \hbar \omega) J_{l}\left(\frac{e V_{\alpha}}{\hbar \omega}\right) .
$$

up to corrections of the order of $\hbar \omega / E_{F}$ which arise from the difference of the wave vectors of the sidebands $p_{l}=\sqrt{2 m(E+l \hbar \omega)} / \hbar$ and the wavevector at energy $E$.
The current operator Eq. (11) is expressed in terms of the incoming (and outgoing) states of the stationary timeindependent scattering problem. Eq. (13) can now be used to find the current operator in terms of the reservoir states $\hat{\mathbf{a}}_{\alpha, n}^{\prime}$. The current operator becomes

$$
\begin{aligned}
\hat{I}_{\alpha}(t)= & \frac{e}{h} \int d E \int d E^{\prime} \sum_{\gamma \delta} \sum_{l k=-\infty}^{\infty}\left(\hat{\mathbf{a}}^{\prime}\right)_{\gamma}^{\dagger}(E-l \hbar \omega) \\
& J_{l}\left(\frac{e V_{\gamma}}{\hbar \omega}\right) J_{k}\left(\frac{e V_{\delta}}{\hbar \omega}\right) e^{i\left(E-E^{\prime}\right) t / \hbar} \\
& \mathbf{A}_{\gamma \delta}\left(\alpha, E, E^{\prime}\right) \hat{\mathbf{a}}_{\delta}^{\prime}\left(E^{\prime}-k \hbar \omega\right)
\end{aligned}
$$

where we have introduced the current matrix [39]

$$
\mathbf{A}_{\delta \gamma}\left(\alpha, E, E^{\prime}\right)=\delta_{\alpha \delta} \delta_{\alpha \gamma} \mathbf{1}_{\alpha}-\mathbf{s}_{\alpha \delta}^{\dagger}(E) \mathbf{s}_{\alpha \gamma}\left(E^{\prime}\right) .
$$

It is assumed that the modulation imposed on the system is so slow that the contacts can still be regarded as being in a dynamic equilibrium state. Thus the quantum statistical average can be found by evaluating averages of the $\hat{\mathbf{a}}_{\alpha}(E-l \hbar \omega)$ as for an equilibrium system. Replacing the $\hat{\mathbf{a}}_{\alpha}(E-l \hbar \omega)$ by their equilibrium statistical expectation values we find,

$$
\begin{aligned}
I_{\alpha}(t)= & \frac{e}{h} \int d E \sum_{\gamma, l k} \operatorname{Tr} \mathbf{A}_{\gamma \gamma}(\alpha, E, E+(k-l) \hbar \omega) \times \\
& J_{l}\left(\frac{e V_{\gamma}}{\hbar \omega}\right) J_{k}\left(\frac{e V_{\gamma}}{\hbar \omega}\right) e^{-i(k-l) \omega t} f_{\gamma}(E-l \hbar \omega) .
\end{aligned}
$$

where $f_{\gamma}(E)=f\left(E-\mu_{\gamma}\right)$ is the Fermi distribution function for contact $\gamma$. Here $\mu_{\gamma}$ is the electrochemical potential of reservoir $\gamma$. In Eq. (16) the trace is over all channels in lead $\alpha$. Taking into account the symmetry properties of the current matrix under exchange of the energy arguments it can be shown that the current given by Eq. (16) is real.

From Eq. (16) we find that for the dc-current only the terms $l=k$ contribute. In this case, as is seen by looking at Eq. (16), the energy arguments of the current matrix are equal. The trace of the current matrix at equal energy arguments and equal lower lead indices are just transmission and reflection probabilities. We define $T_{\alpha \gamma}(E)=-\operatorname{Tr} \mathbf{A}_{\gamma \gamma}(\alpha, E, E)$. For unequal indices $\alpha$ and $\gamma$ this is the transmission probability for carriers incident in lead $\gamma$ to be transmitted into contact $\alpha$. If also $\alpha=\gamma$ the trace of the current matrix is equal to the probability $R_{\alpha \alpha}$ of carriers incident in lead $\alpha$ to be reflected back into lead $\alpha$, minus the number of quantum channels $N_{\alpha}$ at energy $E$. In this notation, particle conservation in the scattering process is expressed by the sum rule $\sum_{\gamma} T_{\alpha \gamma}=0$. For the dc-current we find thus

$$
I_{\alpha}(0)=-\frac{e}{h} \int d E \sum_{\gamma, l} T_{\alpha \gamma}(E) J_{l}^{2}\left(\frac{e V_{\gamma}}{\hbar \omega}\right) f_{\gamma}(E-l \hbar \omega) .
$$

Now we expand in this expression the Bessel functions in powers of the applied oscillating potentials $V_{\gamma}$. The 
zero-th order terms gives the dc-current $I^{d c,(0)}\left[\left\{V_{\beta}\right\}\right]$ that flows as a consequence of stationary differences in the applied potentials. We use a superscript (0) to denote a response to an external potential only. Since the potential in the interior is kept fixed this $I-V$ characteristic is not gauge invariant. A discussion is provided in Ref. [29] and by Christen and one of the authors [35. The next term is second order in the amplitudes of the oscillating voltages. For identifying conductance coefficients recall that the applied potential is of the form $V_{\gamma}(t)=\frac{1}{2} V_{\gamma}(\omega) e^{i \omega t}+\frac{1}{2} V_{\gamma}^{*}(\omega) e^{-i \omega t}$, with the amplitudes taken as real. Thus, from the calculated response to $V_{\gamma}(t)$ we need to extract the response to the Fourier amplitudes. These second order terms are determined by the photo-conductances

$$
\begin{array}{r}
g_{\alpha \beta \gamma}^{(0)}\left[\omega,-\omega ;\left\{V_{\delta}(0)\right\}\right]=-\delta_{\beta \gamma} \frac{e^{3}}{h} \int d E T_{\alpha \beta}\left[E ;\left\{V_{\delta}(0)\right\}\right] \\
\frac{f_{\beta}(E+\hbar \omega)+f_{\beta}(E-\hbar \omega)-2 f_{\beta}(E)}{(\hbar \omega)^{2}} .
\end{array}
$$

The photo-conductance $g_{\alpha \beta \gamma}^{(0)}\left[\omega,-\omega ;\left\{V_{\delta}(0)\right\}\right]$ determines the zero-frequency current in contact $\alpha$ in response to a second order voltage oscillation $V_{\beta}^{2}(\omega)$ at contact $\beta$. Note that the external photo-conductance generated by bilinear products $V_{\beta}(\omega) V_{\gamma}(\omega)$ with $\beta$ unequal to $\gamma$ vanishes. Instead of a second order difference in Fermi functions we can express the photo-conductance as a second order difference of transmission probabilities

$$
\begin{aligned}
g_{\alpha \beta \gamma}^{(0)}[\omega,-\omega] & =-\delta_{\beta \gamma} \frac{e^{3}}{h} \int d E f_{\beta}(E) \times \\
& \frac{T_{\alpha \beta}(E+\hbar \omega)+T_{\alpha \beta}(E-\hbar \omega)-2 T_{\alpha \beta}(E)}{(\hbar \omega)^{2}} .
\end{aligned}
$$

For simplicity we have not explicitly indicated the dependence on the stationary potentials $V_{\delta}(0)$. Eq. (19) shows clearly that we obtain an externally induced photocurrent only if the transmission probabilities through the sample are energy dependent. Thus, for a quantum point contact or for a quantized Hall conductor, where we encounter situations characterized by transmission probabilities which are either zero or one, there is no externally induced photo current. This form of the photoconductance also makes it evident that current conservation is satisfied due to the unitarity of the scattering matrix: The sum of all photo-conductances over all contacts adds up to zero, $\sum_{\alpha} g_{\alpha \beta \gamma}^{(0)}[\omega,-\omega]=0$. However, similar to the dc I-V characteristic these conductances are not gauge invariant. The sum $\sum_{\beta} g_{\alpha \beta \gamma}^{(0)}[\omega,-\omega]$ does not vanish and consequently the photo-current evaluated with these expressions depends not only on voltage differences.

Let us next consider the displacement current. The current at the frequency $\omega$ is determined by the terms in Eq. (16) for which $k-l=1$ and it is given by

$$
I_{\alpha}(\omega)=\frac{e}{h} \int d E \sum_{\gamma, l} \operatorname{Tr} \mathbf{A}_{\gamma \gamma}(\alpha, E, E+\hbar \omega) \times
$$

$$
J_{l}\left(\frac{e V_{\gamma}}{\hbar \omega}\right) J_{l+1}\left(\frac{e V_{\gamma}}{\hbar \omega}\right) f_{\gamma}(E-l \hbar \omega)
$$

Linearising the response to an oscillating external potential yields the admittance previously found [29.30],

$$
\begin{aligned}
g_{\alpha \beta}^{(0)}\left[\omega ;\left\{V_{\gamma}(0)\right\}\right]= & \frac{e^{2}}{h} \int d E \operatorname{Tr} \mathbf{A}_{\beta \beta}\left[\alpha, E, E+\hbar \omega ;\left\{V_{\gamma}(0)\right\}\right] \\
& \times \frac{f_{\beta}(E)-f_{\beta}(E+\hbar \omega)}{\hbar \omega} .
\end{aligned}
$$

The external admittance given by Eq. (21) has been the starting point of a self-consistent discussion of ac transport based on the scattering matrix approach. The approach has been illustrated in a number of works 404 4. The next term in the expansion is third order in the oscillating potentials and will not be needed here.

We remark that the external photo-conductances Eq. (19) are like the dc-current determined by transmission probabilities only. In contrast, the displacement current invokes products of scattering matrices at different energies and thus depends also on the phases of the scattering matrix. Expressed in a more physical language, the displacement current is sensitive to the densities of carriers, expressed here via energy derivatives of phases. Below we find that the self-consistent photo-current contains in fact not only transmission probabilities but, like the displacement current, also information on the charge accumulated in the conductor.

Before considering the effect of screening, we discuss the relation of the external response to previous work. A discussion of shot noise in a conductor with applied ac voltages can be found in Appendix A.

\section{A. Two-terminal conductors}

We consider a two-terminal conductor which consists of a tunneling barrier connected on either side to a large contact. This is the geometry considered by Tien and Gordon [i] and Tucker [2]. The results we obtain from the external response described above are in agreement with these earlier works. Below we emphasize the arbitrary nature of these results arising from the lack of a selfconsistent treatment of the Coulomb interaction. As a consequence, different but physically identical configurations of voltages lead to different results. Later we will show how the results for these two arrangements change when screening is taken into account, for the specific example where the barrier is a resonant tunneling barrier.

First we consider the Tien and Gordon case, where one of the contact potentials is oscillating and the other is kept fixed, $V_{1}(\omega)=V(\omega)$ and $V_{2}(\omega)=0$. For simplicity we assume that the scattering matrix has been diagonalized such that transmission through the barrier is described by a transmission probability $T_{m}(E)$ and a 
reflection probability $R_{m}(E)$ for the m-th eigen channel. Using Eq. (17) and using the sum rule for Bessel functions, $\sum_{l} J_{l+k}(x) J_{l}(x)=\delta_{k 0}$, we find

$$
\begin{aligned}
I_{1}(0)= & -\frac{e}{h} \int d E \sum_{l m} J_{l}^{2}\left(\frac{e V(\omega)}{\hbar \omega}\right) T_{m}(E) \times \\
& \times\left[f_{1}(E+l \hbar \omega)-f_{2}(E)\right] .
\end{aligned}
$$

Tien and Gordon [1] express the transmission probabilities with the help of Bardeen's formula $T=$ $4 \pi|t|^{2} \nu_{1}(E) \nu_{2}(E+l \hbar \omega)$ in terms of a matrix element $t$ and the density of states $\nu_{1}(E)$ and $\nu_{2}(E)$ to the left and right of the barrier. In our work the energy $E$ is a global variable, whereas Tien and Gordon measure energy in the densities of states from the conduction band bottom to the left and right of the barrier.

The time-dependent current was investigated by Tucker for the same geometry [2]. Using Eq. (16) we find

$$
\begin{aligned}
I_{1}(t)= & \sum_{l k \gamma} \operatorname{Tr}_{\gamma \gamma}(\alpha, E, E+k \hbar \omega) \\
& J_{l}\left(\frac{e V(\omega)}{\hbar \omega}\right) J_{l+k}\left(\frac{e V(\omega)}{\hbar \omega}\right) e^{-i k \omega t} \times \\
& \times f_{\gamma}(E-l \hbar \omega) .
\end{aligned}
$$

This is the result of Tucker [2] except that he considers the barrier transmission to be energy independent.

However, the results obtained above are not invariant under an equal shift of all potentials. For example, in the Tien and Gordon case, an experimentally equivalent situation would be to set $V_{1}(\omega)=V(\omega) / 2$ and $V_{2}(\omega)=-V(\omega) / 2$. This, however, yields a different result in the non-interacting theory. Even worse, setting $V_{1}(\omega)=V_{2}(\omega)=V(\omega) / 2$ should yield no photocurrent, but gives the same as for $V_{1}(\omega)=V(\omega) / 2$ and $V_{2}(\omega)=-V(\omega) / 2$. To remedy this we introduce in the next section a simple selfconsistent scheme to achieve charge and current conservation, similar to one used previously 29.43 .

\section{B. Density operator}

When applying voltages to the conductor, the sample will be charged. The net charge of the sample in response to a potential applied to a contact can be decomposed into two contributions: A charge response, called the injectance of the contact, at fixed internal electric potential and a charge response due to an electrically induced potential. Here we determined the injectances of a multiterminal conductor. In the next section these results are used when treating the problem with interactions.

At zero frequency, the number of electrons in the sample is determined by the operator 44.

$$
\begin{aligned}
\hat{N}= & \sum_{\alpha \beta n m} \int d^{2} \mathbf{r} \int d E \nu_{\alpha n}^{1 / 2}(E) \nu_{\beta m}^{1 / 2}(E) \times \\
& \Psi_{\alpha n}^{*}(r, E) \Psi_{\beta m}(r, E) \hat{a}_{\alpha n}^{\dagger}(E) \hat{a}_{\beta m}(E),
\end{aligned}
$$

where $\nu_{\alpha n}(E)$ is the density-of-states for channel $n$ in contact $\alpha$, and $\Psi_{\alpha n}(r, E)$ is the corresponding wavefunction for a scattering state describing carriers incident in contact $\alpha$ in channel $n$.

We now define the partial density-of-states matrix $\frac{d \mathcal{N}_{\alpha \beta}}{d E}$, with elements

$$
\frac{d \mathcal{N}_{\alpha \beta, n m}}{d E}=\int d^{2} \mathbf{r} \nu_{\alpha n}^{1 / 2}(E) \nu_{\beta m}^{1 / 2}(E) \Psi_{\alpha n}^{*}(r, E) \Psi_{\beta m}(r, E) .
$$

This matrix can also be expressed in terms the scattering matrix and its derivatives 44.

$$
\frac{d \mathcal{N}_{\gamma \delta}}{d E}=-\frac{1}{4 \pi i} \sum_{\beta}\left[\mathbf{s}_{\beta \gamma}^{\dagger}(E) \frac{d \mathbf{s}_{\beta \delta}(E)}{d E}-\frac{d \mathbf{s}_{\beta \gamma}^{\dagger}(E)}{d E} \mathbf{s}_{\beta \delta}(E)\right] .
$$

Using this and Eq. (13) we find the number operator in the presence of oscillating contact potentials

$$
\begin{aligned}
\hat{N}= & \sum_{\alpha \beta l k} \int d E J_{l}\left(\frac{e V_{\alpha}}{\hbar \omega}\right) J_{k}\left(\frac{e V_{\beta}}{\hbar \omega}\right) \times \\
& \left(\hat{\mathbf{a}}^{\prime}\right)_{\alpha}^{\dagger}(E-l \hbar \omega) \frac{d \mathcal{N}_{\alpha \beta}}{d E} \hat{\mathbf{a}}_{\beta}^{\prime}(E-k \hbar \omega),
\end{aligned}
$$

with the expectation value

$$
N=\sum_{\alpha l} \int d E J_{l}^{2}\left(\frac{e V_{\alpha}}{\hbar \omega}\right) \operatorname{Tr} \frac{d \mathcal{N}_{\alpha \alpha}}{d E} f_{\alpha}(E-l \hbar \omega)
$$

We can shift the frequency dependence from the Fermi function to the partial density of states

$$
N=\sum_{\alpha} \int d E J_{l}^{2}\left(\frac{e V_{\alpha}}{\hbar \omega}\right) \frac{d N_{\alpha}^{(0)}}{d E} f_{\alpha}(E)
$$

and thus identify the injectance $\frac{d N_{\alpha}^{(0)}}{d E}$ at energy $E$ in the presence of a potential variation at contact $\alpha$. Here the upper index 0 is once more used to emphasize that this density is evaluated at fixed internal potential. To second order in the oscillating potential, $V_{\alpha}(\omega)$, the injectance is

$$
\begin{array}{r}
\frac{d N_{\alpha}^{(0)}}{d E}=\operatorname{Tr}\left[\frac{d \mathcal{N}_{\alpha \alpha}(E)}{d E}-\frac{e^{2}}{2} \frac{\left|V_{\alpha}(\omega)\right|^{2}}{(\hbar \omega)^{2}} \times\right. \\
\left.\left(\frac{d \mathcal{N}_{\alpha \alpha}(E+\hbar \omega)}{d E}+\frac{d \mathcal{N}_{\alpha \alpha}(E-\hbar \omega)}{d E}-2 \frac{d \mathcal{N}_{\alpha \alpha}(E)}{d E}\right)\right]
\end{array}
$$

In the limit that $\left|V_{\alpha}(\omega)\right|$ becomes small compared to $\hbar \omega$ the injectance is that produced by a static voltage. 


\section{INTERNAL RESPONSE: SELF-CONSISTENT SCREENING}

In response to a potential variation at a contact the charge distribution in the interior of the sample is driven away from its equilibrium pattern. Coulomb interactions oppose such a variation. In the problem of interest here a variation of the sample charge can come about both because we in general consider a biased sample such that a dc-current flows and because we subject the sample to ac-voltages. In general it is a non-equilibrium dynamical potential landscape that matters. Here for simplicity we consider the sample to be zero-dimensional and assume that it suffices to consider a single internal potential $U$. Such an approximation is often used in the literature on the Coulomb blockade and in the scattering approach to electrical conduction has been used to discuss the nonlinear I-V characteristic of mesoscopic samples [35] and ac-transport in Refs. 43, 30,45]. At equilibrium, if all voltages at the contact of the sample are equal, and in the absence of ac-potentials, the value of this potential is $U=U_{e q}$. Our first task is to determine the zerofrequency part of this potential.

To be more specific we now consider a sample coupled to a gate, as an example see Fig. 1. We denote the contact to the gate by the index $g$ and the capacitance of the central region of the conductor to the gate by $C_{g}$. The capacitance between the central region of the conductor to the reservoir $\alpha$ is denoted by $C_{\alpha}$. Next we introduce an index $\nu$ which runs over all $N$ current contacts of the sample $\nu=\alpha=1,2, . . N$ and in addition includes the contact to the gate $\nu=N+1=g$.

\section{A. Static internal potential}

Consider first the equilibrium potential $U_{0}^{e q}$. The grandcanonical potential with the Coulomb energy included is minimal for a potential $U_{0}^{e q}$ that obeys the Poisson equation. In our case the Poisson equation is discretisized and is expressed with the help of the geometrical capacitances introduced above. The net electronic charge on the sample is that permitted by the Coulomb interaction:

$$
Q-Q^{+}=\sum_{\nu} C_{\nu}\left(U-V_{\nu}\right)
$$

Here $Q$ is the electronic charge, $Q^{+}$is an effective "ionic charge" created by the donors and $C_{\nu}$ are the geometrical capacitances.

For $V_{\nu}=0$, the equilibrium charge $Q=Q_{0}^{e q}$ and the equilibrium potential $U=U_{0}^{e q}$ follow from Eq. (31) as follows. The electronic charge on the conductor can be expressed as a sum of all the charges injected from the various contacts,

$$
Q_{0}^{e q}=\sum_{\alpha} \int_{-\infty}^{\mu} e \frac{d N_{\alpha}\left(U_{0}^{e q}\right)}{d E} d E
$$

where the injectance [45,33] of contact $\alpha$ is given by Eq. (30). Note that the scattering matrix and thus the injectance also depends on $U_{0}^{e q}$. Eq. (32) is thus a selfconsistent equation for the equilibrium potential.

Next, let us keep the ac-voltages turned of but apply dc voltages to the contacts, charge will flow into the conductor causing a shift of the static potential in the barrier. We denote the resulting potential by $U_{0}$. It is a function of the applied potentials $V_{\nu}$ since now the injected charge depends on all the applied voltages. The injected charge is given by

$$
Q_{0}=\sum_{\alpha} \int_{-\infty}^{\mu_{\alpha}} e \frac{d N_{\alpha}\left(U_{0}\right)}{d E} d E
$$

Using Eq. (32) to express the effective background charge in terms of the scattering matrix and the charges on the capacitors gives the following self-consistent equation for determining the internal static potential in the sample

$$
\begin{aligned}
& \sum_{\alpha} \int_{-\infty}^{\mu_{\alpha}} e \frac{d N_{\alpha}\left(U_{0}\right)}{d E} d E-\int_{-\infty}^{\mu_{e q}} \sum_{\alpha} e \frac{d N_{\alpha}\left(U_{0}^{e q}\right)}{d E} d E \\
& =\sum_{\nu} C_{\nu}\left(U_{0}-V_{\nu}\right) .
\end{aligned}
$$

Here $V_{\alpha}=\mu_{\alpha}-\mu$ is the deviation of the electrochemical potential in contact $\alpha$ from its equilibrium value $\mu$. This approach was used by Christen and Büttiker [35] to study the nonlinear conductance for a resonant tunneling barrier.

Next, consider next the case that is really of interest here. In addition to possible static voltage differences we have time-dependent potentials at the contacts. As a consequence the (unscreened) charge $Q(t)$ in the sample is also a function of time. It can be Fourier transformed, and we expect Fourier components at the oscillation frequency $\omega$ of the voltage and at all higher harmonics, $k \omega$. As a consequence the potential inside the conductor will also oscillate and will similarly have Fourier components at all harmonics, $U(k \omega)$. If an oscillating voltage at a contact, due to non-linear processes, also changes the time-averaged charge in the sample then the potential $U_{0}$ as determined above would be modified by the presence of the oscillating potentials. To take this into account we write the injected charge as a response to external potentials in the presence of a self-consistently determined static potential plus the response from the internal oscillating potential. The response to the internal potential is determined by three unknown response coefficients, $\chi_{i \alpha}$, $\chi_{\alpha i}$ and $\chi_{i i}$ such that,

$$
\begin{aligned}
Q_{0}= & \sum_{\alpha} \int e \frac{d N_{\alpha}^{(0)}}{d E} d E+\sum_{\alpha} \int d E \chi_{i \alpha}(E) U^{*}(\omega) V_{\alpha}(\omega) \\
& +\sum_{\alpha} \int d E \chi_{\alpha i}(E) V_{\alpha}^{*}(\omega) U(\omega)+\chi_{i i}(E)|U(\omega)|^{2} .
\end{aligned}
$$


To determine $\chi_{i \alpha}, \chi_{\alpha i}$ and $\chi_{i i}$ we use the fact that the injectance be invariant under a shift of all oscillating potentials by an equal amount. This yields the coefficients

$$
\begin{aligned}
\chi_{i \alpha}(E)= & \chi_{\alpha i}(E)=\operatorname{Tr}\left[\frac { 1 } { 2 } ( \frac { e } { \hbar \omega } ) ^ { 2 } \left(\frac{d \mathcal{N}_{\alpha \alpha}(E+\hbar \omega)}{d E}\right.\right. \\
& \left.\left.+\frac{d \mathcal{N}_{\alpha \alpha}(E-\hbar \omega)}{d E}-2 \frac{d \mathcal{N}_{\alpha \alpha}(E)}{d E}\right)\right] \\
\chi_{i i}(E)= & -\sum_{\alpha} \chi_{i \alpha}(E) .
\end{aligned}
$$

With this we can express the gauge-invariant injectance as

$$
\begin{gathered}
\frac{d N_{\alpha}}{d E}=\operatorname{Tr}\left[\frac{d \mathcal{N}_{\alpha \alpha}(E)}{d E}-\frac{e^{2}}{2} \frac{\left|V_{\alpha}(\omega)-U(\omega)\right|^{2}}{(\hbar \omega)^{2}} \times\right. \\
\left.\left(\frac{d \mathcal{N}_{\alpha \alpha}(E+\hbar \omega)}{d E}+\frac{d \mathcal{N}_{\alpha \alpha}(E-\hbar \omega)}{d E}-2 \frac{d \mathcal{N}_{\alpha \alpha}(E)}{d E}\right)\right]
\end{gathered}
$$

Eqs. (34) and (38) now allows us to find the static internal potential $U_{0}$ in the presence of static and oscillating contact voltages. Note that $\frac{d N_{\alpha}}{d E}$ depends on $U_{0}$ since the scattering matrix depends on $U_{0}$. The potential $U_{0}$ depends on the dc-voltages applied to the sample and depends through non-linear processes on the amplitudes of the ac-voltages and the frequency.

Our next task is now to find the current response to the oscillating internal potential $U(t)$.

\section{B. dc-current}

Consider first the photo-induced dc-current. The dccurrent can be divided into two parts, one due to direct transmission processes, and one due to transmission after absorption (emission) of a photon followed by its emission (absorption). Both processes take place in a selfconsistently determined electrostatic background, which depends on all voltages at all frequencies.

$$
I_{\alpha}(0)=I_{\alpha}^{d c}\left[\left\{V_{\beta}(0)\right\}\right]+I_{\alpha}^{p h}\left[\left\{V_{\beta}(0)\right\}\right]
$$

Here $I_{\alpha}^{d c}\left[\left\{V_{\beta}(0)\right\}\right]$ is determined from the first term of the sum in Eq. (17), where now the scattering matrix depends on $U_{0}$.

The photo-current can be written generally as the sum of the response to the external oscillating potential and the internal potential $U(\omega)$. To proceed we now consider $\epsilon=e U(\omega) /(\hbar \omega)$, a small parameter in which we can expand. All the oscillating contact potentials are also of order $\epsilon$. In this work we will stop this expansion at the first non-trivial order. Since photon-assisted tunneling is of second or higher order in the oscillating potentials, we carry the expansion to second order.

For the photo-current we obtain

$$
\begin{aligned}
I_{\alpha}^{p h}\left[\left\{V_{\beta}(0)\right\}\right]= & \sum_{\beta \gamma} g_{\alpha \beta \gamma}^{(0)}\left[\omega,-\omega ;\left\{V_{\delta}(0)\right\}\right] V_{\beta}(\omega) V_{\gamma}^{*}(\omega) \\
& +\sum_{\beta} g_{\alpha \beta i}\left[\omega,-\omega ;\left\{V_{\delta}(0)\right\}\right] V_{\beta}(\omega) U^{*}(\omega) \\
& +\sum_{\gamma} g_{\alpha i \gamma}\left[\omega,-\omega ;\left\{V_{\delta}(0)\right\}\right] U(\omega) V_{\gamma}^{*}(\omega) \\
& +g_{\alpha i i}\left[\omega,-\omega ;\left\{V_{\delta}(0)\right\}\right] U(\omega) U^{*}(\omega)
\end{aligned}
$$

where the index $i$ refers to responses due to the internal potential $U(\omega)$.

The responses to the internal potential are found by demanding that the current is invariant with respect to a shift of all voltages (gauge invariance). Lowering all voltages at frequency $\omega$ by $U(\omega)$ shifts the internal potential to the external potentials. Comparing the resulting expression with Eq. (40) gives

$$
\begin{aligned}
& g_{\alpha \beta i}\left[\omega,-\omega ;\left\{V_{\delta}(0)\right\}\right]=-\sum_{\gamma} g_{\alpha \beta \gamma}^{(0)}\left[\omega,-\omega ;\left\{V_{\delta}(0)\right\}\right], \\
& g_{\alpha i \gamma}\left[\omega,-\omega ;\left\{V_{\delta}(0)\right\}\right]=-\sum_{\beta} g_{\alpha \beta \gamma}^{(0)}\left[\omega,-\omega ;\left\{V_{\delta}(0)\right\}\right], \\
& g_{\alpha i i}\left[\omega,-\omega ;\left\{V_{\delta}(0)\right\}\right]=\sum_{\beta \gamma} g_{\alpha \beta \gamma}^{(0)}\left[\omega,-\omega ;\left\{V_{\delta}(0)\right\}\right] .
\end{aligned}
$$

Thus the photo-responses to the internal potential are determined by combinations of external photoconductances.

With these conductances, the dc-current can be written as

$$
\begin{aligned}
I_{\alpha}(0)= & I_{\alpha}^{d c}\left[\left\{V_{\beta}(0)\right\}\right] \\
& +\sum_{\beta} g_{\alpha \beta \beta}^{(0)}\left[\omega,-\omega ;\left\{V_{\gamma}(0)\right\}\right]\left|V_{\beta}(\omega)-U(\omega)\right|^{2} .
\end{aligned}
$$

Note that the current depends on the difference between an applied voltage and the internal voltage only.

All the non-linear transport coefficients in Eqs. (40 44) also depend on $U_{0}$, the self-consistent dc-potential.

\section{Displacement current}

The current at frequency $\omega$ is only needed to first order in the applied oscillating voltages. In addition to the external potential the oscillating internal potential also contributes to the current. In the presence of the internal potential the general form for the current is to first order in the potentials

$$
\begin{aligned}
I_{\alpha}(\omega)= & \sum_{\beta} g_{\alpha \beta}^{(0)}\left[\omega ;\left\{V_{\gamma}(0)\right\}\right] V_{\beta}(\omega) \\
& +g_{\alpha i}\left[\omega ;\left\{V_{\gamma}(0)\right\}\right] U(\omega) .
\end{aligned}
$$

Here $g_{\alpha \beta}^{(0)}\left[\omega ;\left\{V_{\gamma}(0)\right\}\right]$ are the external ac-conductances given by Eq. (21) and $g_{\alpha i}$ is the ac-response to the internal potential. Again we determine $g_{\alpha i}$ through the requirement that this expression is invariant under an 
overall shift of the potential. This gauge invariance argument determines the response to the internal potential in terms of external responses; $g_{\alpha i}\left[\omega ;\left\{V_{\gamma}(0)\right\}\right]=$ $-\sum_{\beta} g_{\alpha \beta}^{(0)}\left[\omega ;\left\{V_{\gamma}(0)\right\}\right]$.

Both for the dc-current and the ac-current we now know the response to the external voltages $V_{\gamma}(0)$ and to the internal potential $U(t)$. But the internal potential is thus far not determined. This is our next task.

To be more specific we now return to the sample shown in Fig. 11. The current at contact $\alpha$ is the particle current plus the displacement current (capacitive) current $I_{\alpha}(\omega)-i \omega C_{\alpha}\left(V_{\alpha}(\omega)-U(\omega)\right)$ with $I_{\alpha}$ as determined above. The current from the gate to the sample is purely capacitive and is given by $I_{g}(\omega)=-i \omega\left(V_{g}(\omega)-U(\omega)\right)$. Since the overall charge at frequency $\omega$ is conserved the sum of these currents must vanish. Thus we must have

$$
\sum_{\alpha} I_{\alpha}(\omega)=-i \omega \sum_{\nu} C_{\nu}\left(U(\omega)-V_{\nu}(\omega)\right) .
$$

Solving this equation for the internal potential yields

$$
U(\omega)=\frac{\sum_{\alpha \beta} g_{\alpha \beta}^{(0)}\left[\omega ;\left\{V_{\gamma}(0)\right\}\right] V_{\beta}(\omega)-i \omega \sum_{\nu} C_{\nu} V_{\nu}(\omega)}{\sum_{\alpha \beta} g_{\alpha \beta}^{(0)}\left[\omega ;\left\{V_{\gamma}(0)\right\}\right]-i \omega \sum_{\nu} C_{\nu}} .
$$

The external ac-conductances and the geometrical capacitances determine the potential $U(\omega)$ and determine the self-consistent dc-current due to photo-assisted tunneling and the self-consistent ac-conductances.

\section{RESONANT TUNNELING BARRIER}

As an application of the selfconsistent theory developed above, we consider the photo-induced dc-current through a resonant tunneling barrier. The experimental setup is taken as sketched in Fig. 11. Each side of the resonant barrier is connected to reservoirs with chemical potentials $\mu_{1}$ and $\mu_{2}$ and capacitances $C_{1}$ and $C_{2}$. The interior of the barrier is coupled to a gate with a capacitance $C_{g}$. For simplicity we assume that the gates are macroscopic with no dynamics of their own. A dc bias will be applied by making $e V \equiv e\left(V_{1}-V_{2}\right)=\mu_{1}-\mu_{2}$ non-zero.

The scattering matrix close to a resonance is given by the Breit-Wigner formula 46 48

$$
s_{m n}=\left[\delta_{m n}-i \frac{\sqrt{\gamma_{m} \gamma_{n}}}{E-E_{0}-e U_{0}+i \gamma / 2}\right] e^{i\left(\delta_{m}+\delta_{n}\right)} .
$$

Here $\gamma_{n}, n=1,2$ are the partial widths of the resonance proportional to the tunneling probability through the left and right barrier and $\gamma=\sum_{n} \gamma_{n}$ is the total width of the resonance. $\delta_{m}$ are the phases acquired in the reflection or transmission process and $E_{0}$ is the position of the resonance. The term $e U_{0}=e \frac{V_{1}(0)+V_{2}(0)}{2}+W$ ensures invariance upon a shift of the dc voltages [35]. $W$ is determined by the condition Eq. (34) and Eq. (38), and is a function of $V_{1}(0)-V_{2}(0)$ only. The injectivities are 450

$$
\frac{d N_{\alpha}}{d E}=\frac{1}{2 \pi} \frac{\gamma_{\alpha}}{\left(E-E_{0}-e U_{0}\right)^{2}+(\gamma / 2)^{2}} .
$$

The Breit-Wigner formula is a reasonable form for the scattering matrix as long as the energy does not get close to the next resonance level. Assuming that the level spacing of our system is large enough such that neighboring levels can safely be ignored we will use the formula in a wide energy range.

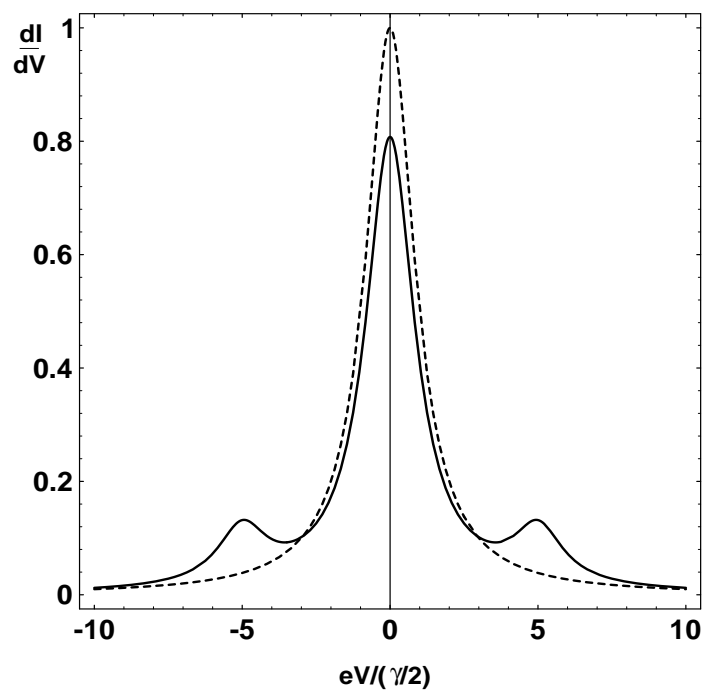

FIG. 2. Differential conductance as a function of dc bias from the non-interacting discussion. The left contact potential is oscillating. The parameters are $\hbar \omega /(\gamma / 2)=5, \epsilon^{2}=0.1$, and the Fermi energy is equal to the resonant energy $\mu=E_{0}$. For comparison the dashed line shows the transmission probability determined from the Breit-Wigner expression.

Photon-assisted tunneling is most easily seen either in the differential conductance as function of bias voltage $d I(V) / d V$, where side-peaks shows up at multiples of the photon energy, or in the dc-current when varying the gate potential [5] $I\left(V_{g}\right)$. In Fig. 2] we show an example of a $d I(V) / d V$-curve using the non-interacting discussion, Eq. (22) and using the Breit-Wigner expression Eq. (48) with $U_{0}=0$. The potential of the left contact oscillates. We apply a dc voltage $V \equiv V_{1}-V_{2}$, take $\hbar \omega /(\gamma / 2)=5$ and consider the symmetric case $\gamma_{1}=\gamma_{2}=\gamma / 2$. In this and all the following examples we use $\epsilon^{2}=0.1$, for which the expansion to second order is pertinent. For this choice of parameters only the first side band peaks can be resolved. In a non-interacting discussion one identifies $U_{0}=V_{g}$ and as a consequence both the differential 
conductance as a function of dc-voltage, or the current as a function of gate voltage, the side bands are observed at a voltage corresponding to the photon energy. Discussions which neglect interactions do not discriminate between these two methods for observing photo-assisted transport. If we now consider the physically meaningful result, the theory which includes interactions, the general behavior will remain the same, but the two methods of analyzing photo-assisted transport, i. e. considering $d I(V) / d V$ or $I\left(V_{g}\right)$ now give in general different results. The effects brought about by screening, discussed in more detail in the next section, are: First, the relative weight of the sidebands and the central peaks will not be the same in the two situations. Second screening also brings about an asymmetry in the weights of the side bands for $\pm n \hbar \omega$. In a discussion that neglects interaction the side bands have the same weight. In contrast, in the interacting case, if the equilibrium chemical potential does not coincide with the resonant energy, screening will be different for the two voltages where peaks are seen, and accordingly their weights will differ. Such asymmetries are seen in experiments [7]. Below we discuss these effects in detail.

\section{A. Gate driven case}

First, consider a sample subject to a dc bias $V=$ $V_{1}-V_{2}$ and an oscillating voltage $V_{g}(\omega)$ applied solely at the gate. For simplicity we take $C_{1}=C_{2}=0$. In this case there can be no dc photo-current when $\mu_{1}=\mu_{2}$, since $\sum_{\beta} \mathbf{A}_{\beta \beta}(\alpha, E, E)=\mathbf{0}$ (see Eqs. (18) and (44)) as a consequence of the unitarity of the scattering matrix. The effect of photon-assisted tunneling in this setup is controlled by the internal potential. Thus, it is of interest to understand how it relates to the applied gate voltage in the presence of screening. From the selfconsistent theory (see Eq. (47)) we find for the ratio of the applied to the external potential

$$
\frac{U(\omega)}{V_{g}(\omega)}=\left[1+\frac{i}{\omega C} \sum_{\alpha \beta} g_{\alpha \beta}^{(0)}(\omega ; V)\right]^{-1}
$$

This ratio is determined by the ac-conductances $g_{\alpha \beta}^{(0)}(\omega ; V)$. These ac-conductances are known. At zero temperature, for the symmetric resonant tunneling barrier $\gamma_{1}=\gamma_{2}$ they are given by $\mathrm{Fu}$ and Dudley [49] and for the asymmetric case $\gamma_{1} \neq \gamma_{2}$ by Büttiker and Christen 33:

$$
\begin{aligned}
g_{11}^{(0)}(\omega) & =g_{21}^{(0)}(\omega)\left[\frac{\gamma_{1}}{\gamma_{2}}-\frac{\gamma}{\gamma_{2}}\left(1-i \frac{\hbar \omega}{\gamma}\right)\right], \\
g_{22}^{(0)}(\omega) & =g_{12}^{(0)}(\omega)\left[\frac{\gamma_{2}}{\gamma_{1}}-\frac{\gamma}{\gamma_{1}}\left(1-i \frac{\hbar \omega}{\gamma}\right)\right], \\
g_{21}^{(0)}(\omega ; V) & =g_{12}^{(0)}(\omega ;-V),
\end{aligned}
$$

$$
\begin{aligned}
g_{12}^{(0)}(\omega) & =\frac{e^{2}}{h} \frac{\gamma_{1} \gamma_{2}}{\gamma \hbar \omega} \frac{1}{1-i \frac{\hbar \omega}{\gamma}} \times \\
\times & {\left[\frac{i}{2} \ln \frac{\left[\left(\mu-\hbar \omega-E_{0}-W-e V / 2\right)^{2}+(\gamma / 2)^{2}\right]}{\left[\left(\mu-E_{0}-W-e V / 2\right)^{2}+(\gamma / 2)^{2}\right]}\right.} \\
+ & \frac{i}{2} \ln \frac{\left[\left(\mu+\hbar \omega-E_{0}-W-e V / 2\right)^{2}+(\gamma / 2)^{2}\right]}{\left[\left(\mu-E_{0}-W-e V / 2\right)^{2}+(\gamma / 2)^{2}\right]} \\
+ & \arctan \left(\frac{\mu+\hbar \omega-E_{0}-W-e V / 2}{\gamma / 2}\right) \\
- & \left.\arctan \left(\frac{\mu-\hbar \omega-E_{0}-W-e V / 2}{\gamma / 2}\right)\right] .
\end{aligned}
$$

With these expressions Eq. (50), the ratio of internal to external potential, can be evaluated. This ratio has two simple limits. In the non-interacting limit $C \rightarrow \infty$, the internal potential directly follows the applied potential. In the limit $C \rightarrow 0$, we have a charge neutral sample and $U(\omega)=0$.

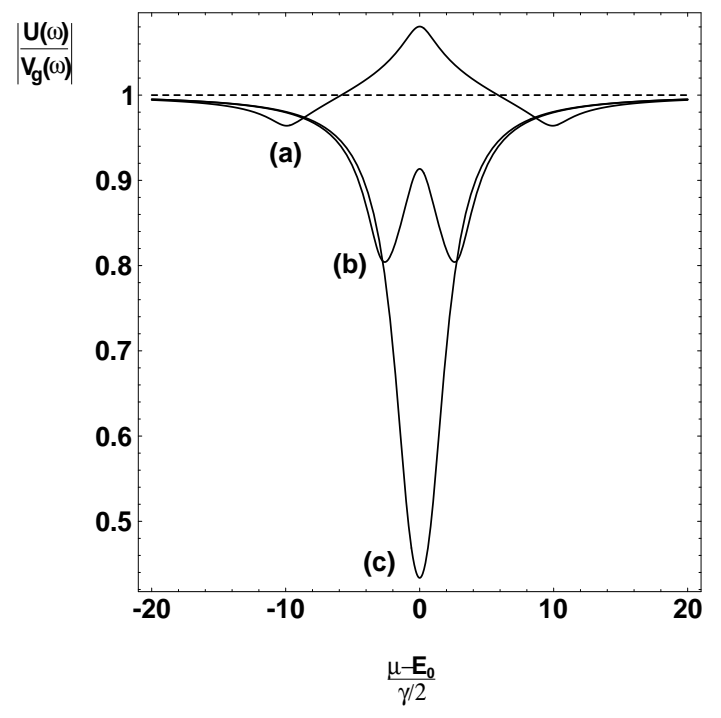

FIG. 3. Ratio of the internal potential to the gate voltage as function of the Fermi energy, for $C=\frac{e^{2}}{\pi \gamma}, V=0$ and for the frequencies $a) \hbar \omega /(\gamma / 2)=10, b) \hbar \omega /(\gamma / 2)=3$, and c) $\hbar \omega /(\gamma / 2)=1$.

In Fig. 3, we show the absolute square ratio of the internal to the external potential for different frequencies, when sweeping the Fermi level through the resonance. The non-screened case $C \rightarrow \infty$, where the ratio is 1 , is shown as the dashed line. It is evident that screening introduces a large renormalisation of the internal potential for this choice of capacitance with a strong dependence on frequency. One observes the largest effect when the Fermi energy is close to the resonance. This is expected since the density in the barrier is a Lorentzian with a peak at resonance 46], thus providing more screening 
electrons. As a function of frequency the ratio changes qualitatively; for low frequencies the internal potential is reduced compared to the external potential, whereas with increasing frequency the situation reverses.

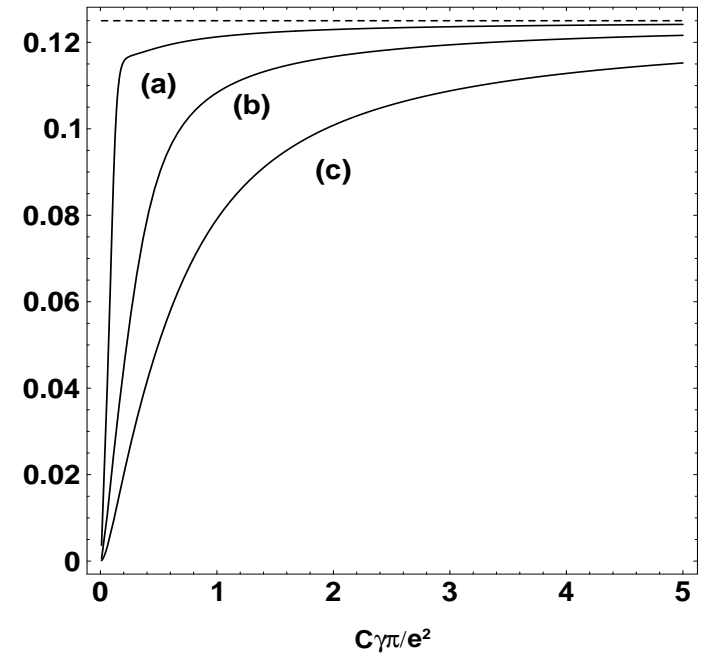

FIG. 4. Ratio of the sideband weight to central peak weight as function of capacitance in the current versus gate voltage characteristic $I\left(V_{g}\right)$ for frequencies $\left.a\right) \hbar \omega /(\gamma / 2)=3$, b) $\hbar \omega /(\gamma / 2)=5$, and $c) \hbar \omega /(\gamma / 2)=10$, when $\epsilon^{2}=0.1$. The dashed line shows the result when no screening is present.

Next consider a the current as a function of gate voltage. Since screening depends on the position of the resonant level compared to the equilibrium electro-chemical potential, the central peak and the sideband will experience a different degree of screening and, thus, their weights will no longer be given by a Bessel function behaviour as in the non-interacting theory. In Fig. At the ratio of the sideband peak to the central peak is shown. The non-interacting theory predicts a ratio of 0.125 for the parameters chosen (dashed line). It is seen that, depending on capacitance and frequency, this ratio can be quite different.

Similarly, when measuring the differential conductance as function of dc voltage screening will also vary as function of voltage. In this case the sideband weight to central peak weight ratio is shown in Fig. 5. Again, large differences with respect to the non-interacting case is possible.

An interesting effect due to the dependence of screening on the dc voltage (or the gate voltage) is that sidebands will no longer be strictly Lorentzian, but skewed. However, this skewing effect is rather small and probably difficult to resolve experimentally.

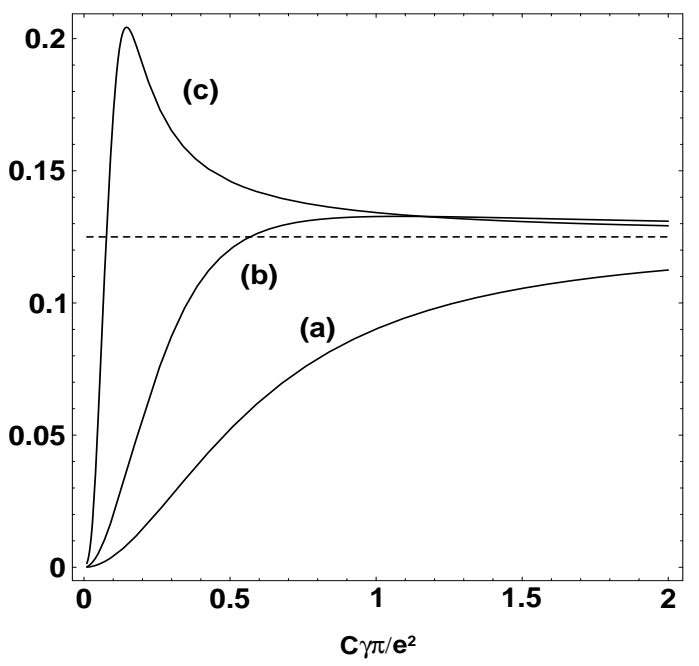

FIG. 5. Ratio of the sideband weight to the central peak weight as function of the the capacitance in the differential conductance $d I / d V$ for the frequencies a) $\hbar \omega /(\gamma / 2)=3$, b) $\hbar \omega /(\gamma / 2)=5$, and $c) \hbar \omega /(\gamma / 2)=10$ and $\epsilon^{2}=0.1$. The dashed line shows the result when no screening is present.

When the Fermi level is off resonance the first sidebands corresponding to absorbing and afterwards emitting a photon and visa versa occur at different potentials. Screening will therefore occur asymmetrically for the two peaks introducing an asymmetry between the \pm sidebands. This effect is illustrated in Fig. 6. Experimental observation of this effect has already been made [7], although it has not been studied systematically.

Another effect is visible in the inset in Fig. 6. One notices that the width of the central peak is significantly larger than the width of the sidebands. Since the capacitance in this example is rather small, the charging energy is large, and when increasing the dc bias voltage the added charge gives rise to a huge increase in the static internal potential. The result is that the resonance floats upwards in energy, widening the peak. For the same reason, the distance from the central peak to the sideband is no longer simply $\hbar \omega$, but substantially larger. 


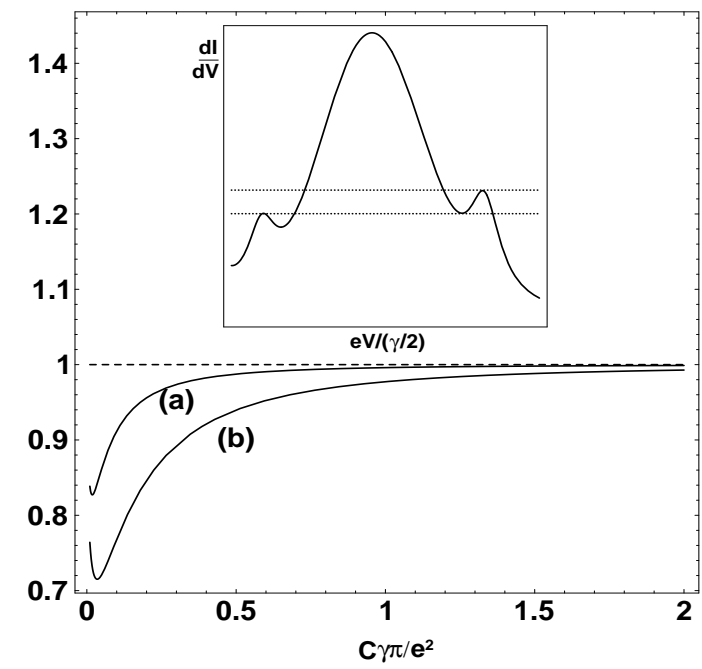

FIG. 6. Weight asymmetry for the $\pm \hbar \omega$ sidebands as function of capacitance in the $d I / d V$-characteristic for $\gamma_{1} /(\gamma / 2)=1 / 4$ and $\gamma_{2} /(\gamma / 2)=3 / 4$, and for a) $\hbar \omega /(\gamma / 2)=3, \mu /(\gamma / 2)=-5$, and $b) \hbar \omega /(\gamma / 2)=5$, $\mu /(\gamma / 2)=-10$. The dashed line shows the result when no screening is present. The insert shows an example of a differential conductance curve as a function of the bias voltage for $\gamma_{1}=\gamma_{2}, \hbar \omega /(\gamma / 2)=5, \mu /(\gamma / 2)=-10$, and $C=0.1 \frac{e^{2}}{\pi \gamma}$. The dashed line is the result without screening.

\section{B. Contact driven case}

A setup often used experimentally is to couple the oscillating field to the conductor via a bowtie antenna [7]. In this case we assume that there is no gate, $C_{g}=0$. For simplicity we take the capacitances across each tunneling barrier to be identical, $C_{1}=C_{2}=C / 2$. The dc-current into contact 1 is then

$$
\begin{aligned}
I_{1}(0 ; V)= & {\left[g_{111}^{(0)}(\omega,-\omega ; V)\left|\frac{\sum_{\alpha} g_{\alpha 2}^{(0)}(\omega ; V)+i \omega C / 2}{\sum_{\alpha \beta} g_{\alpha \beta}^{(0)}(\omega ; V)-i \omega C}\right|^{2}\right.} \\
& \left.+g_{122}^{(0)}(\omega,-\omega ; V)\left|\frac{\sum_{\alpha} g_{\alpha 1}^{(0)}(\omega ; V)+i \omega C / 2}{\sum_{\alpha \beta} g_{\alpha \beta}^{(0)}(\omega ; V)-i \omega C}\right|^{2}\right] \times \\
& \left|V_{2}(\omega)-V_{1}(\omega)\right|^{2} .
\end{aligned}
$$

For the symmetric case $\gamma_{1}=\gamma_{2}$ the dc current will vanish for zero bias, because of the symmetry of the problem. However, in contrast to the gate driven case a zerobias current can be generated for the asymmetric sample, given by

$$
I_{1}(0 ; V=0)=g_{111}^{(0)}(\omega,-\omega)\left|V_{2}(\omega)-V_{1}(\omega)\right|^{2} \frac{\gamma_{2}-\gamma_{1}}{\gamma}
$$

$$
\frac{\left|\frac{g_{12}^{(0)}(\omega)}{\gamma_{1} \gamma_{2}}\right|^{2}+\omega C \frac{\operatorname{Re}\left\{g_{12}^{(0)}(\omega)\right\}}{\gamma_{1} \gamma_{2}}}{\left|\frac{g_{12}^{(0)}(\omega)}{\gamma_{1} \gamma_{2}}-\frac{\omega C}{\hbar \gamma}\right|^{2}}
$$

Since $g_{12}^{(0)}(\omega) /\left(\gamma_{1} \gamma_{2}\right)$ is a function only of $\gamma$ we find that the zero bias current is proportional to the effective asymmetry of the double barrier, $\frac{\gamma_{1}-\gamma_{2}}{\gamma}$. For small capacitances, $C \gamma_{1} \gamma_{2} /\left(2 \hbar g_{12}^{(0)}(\omega)\right) \ll \gamma_{1}, \gamma_{2}$, the current is directly proportional to the asymmetry of the barrier without any renormalisation from screening $I_{1}(0 ; V=$ $0)=g_{111}(\omega,-\omega)\left|V_{2}(\omega)-V_{1}(\omega)\right|^{2} \frac{\gamma_{2}-\gamma_{1}}{\gamma}$. Thus, the acfield effectively pumps electrons through the system. The noninteracting Tien and Gordon discussion, as given by Eq. (22), also predicts a current at zero bias. The result, however, being independent of the asymmetry of the system. This prediction of a zero-bias current for the symmetric case is due to the violations of gaugeinvariance. That a symmetric structure, in the absence of dc-voltages, cannot exhibit a photo-current, can be understood from the following symmetry and invariance conditions. Consider first a variation of the voltage at the left contact $V_{1}(\omega)=V_{0} \cos (\omega t)$ and suppose this produces a dc-photo current $I_{1}$. Then consider a voltage variation of the right contact $V_{2}(\omega)=-V_{0} \cos (\omega t)=$ $V_{0} \cos (\omega t+\pi)$. By symmetry this must give a current $I_{2}=-I_{1}$. In reality, however, due to gauge invariance these two voltage oscillations are experimentally the same and hence must give rise to the same dc-current. But the only dc-current which reflects this symmetry is $I=0$. Clearly, the correct answer is a consequence of gauge invariance.

\section{CONCLUSION}

We have extended the scattering matrix approach to transport in phase-coherent conductors to take into account oscillating contact potentials and internal potentials in nonlinear order. The effect of screening has been taken into account to second order in the oscillating potentials by means of an RPA treatment. The result is a theory, valid for arbitrary dc voltages, which is current and charge conserving (gauge invariant). The internal potential in the conductor has been treated as a single parameter. Certainly, to go beyond this approximation and treat a more realistic continuous potential distribution would be interesting. But even for the case of linear ac-transport, a scattering matrix for continuous potentials exists only to linear order in frequency [31], and exceptionally to second order [44]. Discussions of the dynamic conductance of a ballistic wire over a wide range of frequencies, taking into account spatial potential variations [32, are not yet formulated within the scattering approach. It would also be interesting to extend our discussion to higher order in the applied voltages. 
For large field strengths it is possible to make one of the Bessel functions zero, giving rising to dynamic localization [50,8, 12, Since we find that Bessel functions can in general not give a gauge invariant answer it is clear that the criteria for dynamic localization will be changed in an essential way in the presence of interactions.

We have applied our theory to photon-assisted tunneling using a resonant tunneling barrier as an example. The two standard setups for photon-assisted tunneling, applying the modulation to one of the contacts in a twoterminal experiment, or coupling the potential to the conductor via a gate was examined within the self-consistent theory. In both cases, the inclusion of screening leads to a renormalisation of the non-interacting answer. The driving field is not the applied field but the total field. Since the effective field is dependent on screening and therefore on applied bias, chemical potential etc., the weights of the central peak and the side peaks in the differential conduction versus applied voltage differ from the non-interacting theory. Furthermore, the peak weight is no longer distributed according the increasing order of Bessel functions. This leads to the peak ratios being a complicated function of the screening properties of the system, and predicts an asymmetry between the corresponding left and right sidebands. Asymmetric photoconductance peaks have been observed [7,5].

The necessity to include screening in the treatment of photo-assisted transport is most clearly exemplified by the following consideration. For a spatially symmetric system a non-interacting theory (Tien and Gordon) predicts a photo-current in response to the oscillation of either the left or the right contact voltage. In contrast, the gauge invariant discussion presented here, predicts that a symmetrical system exhibits no photo-current. Our result for the two-terminal resonant tunneling barrier, Eq. (56) is a photo-current which is proportional to the asymmetry of the tunneling rates of the resonant double barrier structure.
In this work we have emphasized that interaction effects are important whenever a variation of a parameter, an oscillation of a voltage, changes the charge away from its equilibrium value. In photo-assisted tunneling it is not sufficient to consider just the dc-current, but a theoretical discussion has to be self-consistent at all frequencies. Thus there is necessarily a relation between the photoassisted dc-current and the displacement current. Only if the charge is investigated at all frequencies can an electrically meaningful, that is gauge invariant, answer be found.

\section{ACKNOWLEDGMENTS}

We are greatful for valuable discussion with Harry Thomas and Anna Prêtre, who helped to clarify the derivation presented in section II.

This work was supported by the Swiss National Science Foundation.

\section{APPENDIX A: CURRENT NOISE}

The analysis of this paper concentrates on the average zero-frequency photo-current. However, the approach used here also allows to find the fluctuations of the current. Of particular interest are the current-current correlations which determine the spectral densities of the current fluctuations. Here we present the general result for the noise spectra of a multi-terminal conductor in the presence of oscillating contact potentials assuming that the internal potential is kept fixed. As with the average dc current a physically meaningful result requires in general a discussion of the effects of screening.

For a multi-probe conductor with potentials $V_{\alpha} \cos (\omega t)$ at frequency $\omega$ applied to the contacts, using Eq. (14), we find the correlation function

$$
\begin{aligned}
\left\langle\left\{\Delta \hat{I}_{\alpha}(t+\tau), \Delta \hat{I}_{\beta}(t)\right\}\right\rangle= & \left(\frac{e}{h}\right)^{2} \int d E d E^{\prime} \sum_{\gamma \delta, l k l^{\prime} k^{\prime}} J_{l}\left(\frac{e V_{\gamma}}{\hbar \omega}\right) J_{k}\left(\frac{e V_{\delta}}{\hbar \omega}\right) J_{l^{\prime}}\left(\frac{e V_{\delta}}{\hbar \omega}\right) J_{k^{\prime}}\left(\frac{e V_{\gamma}}{\hbar \omega}\right) e^{i \frac{E-E^{\prime}}{\hbar} \tau} e^{i\left(l+l^{\prime}-k-k^{\prime}\right) \omega t} \\
& \operatorname{Tr}\left[\mathbf{A}_{\gamma \delta}\left(\alpha, E, E^{\prime}\right) \mathbf{A}_{\delta \gamma}\left(\beta, E^{\prime}+\left(l^{\prime}-k\right) \hbar \omega, E+\left(k^{\prime}-l\right) \hbar \omega\right)\right] \\
& {\left[f_{\gamma}(E-l \hbar \omega)\left(1-f_{\delta}\left(E^{\prime}-k \hbar \omega\right)\right)+f_{\delta}\left(E^{\prime}-k \hbar \omega\right)\left(1-f_{\gamma}(E-l \hbar \omega)\right)\right] . }
\end{aligned}
$$

Here the brackets $\{$,$\} denote the anti-commutator. In$ the presence of ac voltages the current-correlation function is not only a function of the relative time $\tau$ but depends also on the absolute time $t$. Experimentally what is of interest is the noise spectrum on a time scale long compared to $2 \pi / \omega$. Therefore we define the noise spectrum as an average

$$
S_{\alpha \beta}(\tau)=\frac{1}{2 T} \int_{0}^{T} d t\left\langle\left\{\Delta \hat{I}_{\alpha}(t+\tau), \Delta \hat{I}_{\beta}(t)\right\}\right\rangle,
$$

where $T=2 \pi / \omega$ is the period. The factor $1 / 2$ arises because we have symmetrized the correlation function. The spectral density is related to the currentcurrent correlation function via $2 \pi S_{\alpha \beta}(\Omega ; \omega) \delta\left(\Omega+\Omega^{\prime}\right)=$ $(1 / 2)\left\langle\left\{\Delta \hat{I}_{\alpha}(\Omega), \Delta \hat{I}_{\beta}\left(\Omega^{\prime}\right)\right\}\right\rangle$, which is just the Fourier transform of $S(\tau)$. We find 


$$
\begin{aligned}
S_{\alpha \beta}(\Omega ; \omega)= & \left(\frac{e}{\hbar}\right)^{2} \int d E \sum_{\gamma \delta, l k k^{\prime}} J_{l}\left(\frac{e V_{\gamma}}{\hbar \omega}\right) J_{k}\left(\frac{e V_{\delta}}{\hbar \omega}\right) J_{k^{\prime}+k-l}\left(\frac{e V_{\delta}}{\hbar \omega}\right) J_{k^{\prime}}\left(\frac{e V_{\gamma}}{\hbar \omega}\right) \\
& \operatorname{Tr}\left[\mathbf{A}_{\gamma \delta}(\alpha, E, E+\hbar \Omega) \mathbf{A}_{\delta \gamma}\left(\beta, E+\hbar \Omega+\left(k^{\prime}-l\right) \hbar \omega, E+\left(k^{\prime}-l\right) \hbar \omega\right)\right] \\
& {\left[f_{\gamma}(E-l \hbar \omega)\left(1-f_{\delta}(E+\hbar \Omega-k \hbar \omega)\right)+f_{\delta}(E+\hbar \Omega-k \hbar \omega)\left(1-f_{\gamma}(E-l \hbar \omega)\right)\right] . }
\end{aligned}
$$

In the limit of vanishing driving frequency, $\omega=0$, Eq. (3) reduces to the frequency-dependent noise spectra of Ref. [39].

For the special case that the scattering matrices can be taken to be independent of energy, i.e. $\mathbf{A}_{\gamma \delta}(\alpha, E, E+\hbar \omega)=$ $\mathbf{A}_{\gamma \delta}(\alpha)$ Eq. (3) simplifies considerably. Using the addition theorem for Bessel functions we find

$$
\begin{aligned}
S_{\alpha \beta}(0 ; \omega)= & \left(\frac{e}{\hbar}\right)^{2} \int d E \sum_{\gamma \delta, l} \operatorname{Tr}\left[\mathbf{A}_{\gamma \delta}(\alpha) \mathbf{A}_{\delta \gamma}(\beta)\right] J_{l}^{2}\left(\frac{e\left(V_{\delta}-V_{\gamma}\right)}{\hbar \omega}\right) \times \\
& {\left[f_{\gamma}(E+l \hbar \omega)\left(1-f_{\delta}(E)\right)+f_{\delta}(E)\left(1-f_{\gamma}(E+l \hbar \omega)\right)\right] . }
\end{aligned}
$$

For a two-terminal conductor this result is identical to that of Lesovik and Levitov [25] even though in that work this result was derived in response to an electric field and not as here as a response to an oscillating contact voltage. In the experiment of Schoelkopf et al. [26] the shot noise is measured in the presence of an oscillating voltage applied to the contacts of the sample.

We emphasize that the noise spectra given by Eqs. (3) and (田) give only the noise for fixed internal potential. We have already remarked that the average dc current exhibits an external response due to photo-assisted transport only if the transmission probabilities exhibit an energy dependence (see Eq. (19)). In contrast, in the shotnoise spectra, we have an effect even if the scattering matrix is taken to be energy independent. That is a consequence of the fact that the noise spectra depend in a non-linear way on the Fermi functions.

[1] P. K. Tien and J. P. Gordon, Phys. Rev. 129, 647 (1963).

[2] J. R. Tucker, IEEE J. Quantum Elec. 15, 1234 (1979).

[3] M. Büttiker and R. Landauer, Phys. Rev. Lett. 49, 1739 (1982); Phys. Scr. 32, 429 (1985).

[4] C. Bruder and H. Schoeller, Phys. Rev. Lett. 72, 1076 (1994).

[5] L. P. Kouwenhoven, S. Jauhar, J. Orenstein, P. L. McEuen, Y. Nagamune, J. Motohisa, and H. Sakaki, Phys. Rev. Lett. 73, 3443 (1994); L. P. Kouwenhoven, S. Jauhar, K. McCormick, D. Dixon, P. L. McEuen, Yu. V. Nazarov, N. C. van der Vaart, and C. T. Foxon, Phys. Rev. B 50, 2019 (1994); T. H. Oosterkamp, L. P. Kouwenhoven, A. E. A. Koolen, N. C. van der Vaart, and C. J. P. M. Harmans, Phys. Rev. Lett. 78, 1536 (1997).

[6] P. S. S. Guimarães, B. J. Keay, J. P. Kaminski, S. J. Allen, Jr., P. F. Hopkins, A. C. Gossard, L. T. Florez, and J. P. Harbison, Phys. Rev. Lett. 70, 3792 (1993).

[7] H. Drexler, J. S. Scott, S. J. Allen, K. L. Campman, and
A. C. Gossard, Appl. Phys. Lett. 67, 2816 (1995).

[8] B. J. Keay, S. J. Allen, Jr., J. Galán, J. P. Kaminski, K. L. Campman, A. C. Gossard, U. Bhattacharya, and M. J. W. Rodwell, Phys. Rev. Lett. 75, 4098 (1995); B. J. Keay, S. Zeuner, S. J. Allen, Jr., K. D. Maranowski, A. C. Gossard, U. Bhattacharya, and M. J. W. Rodwell, Phys. Rev. Lett. 75, 4102 (1995).

[9] S. Datta and M. P. Anantram, Phys. Rev. B 45, 13761 (1992).

[10] A.-P. Jauho, N. S. Wingreen, and Y. Meir, Phys. Rev. B 50, 5528 (1994); N. S. Wingreen, A.-P. Jauho, and Y. Meir, Phys. Rev. B 48, 8487 (1993).

[11] A. Wacker, A.-P. Jauho, S. Zeuner, and S. J. Allen, Phys. Rev. B 56, 13268 (1997).

[12] M. Wagner, Phys. Rev. A 51, 798 (1995).

[13] M. Wagner, Phys. Rev. Lett. 76, 4010 (1996); M. Wagner and W. Zwerger, Phys. Rev. B 55, R10217 (1997).

[14] R. Agaudo, J. Iñarrea, and G. Platero, Phys. Rev. B 53, 10030 (1996).

[15] R. Aguado and G. Platero, Phys. Rev. B 55, 12860 (1997).

[16] Y. Goldin and Y. Avishai, Phys. Rev. B 55, 16359 (1997).

[17] K. Flensberg, Phys. Rev. B 55, 13118 (1997).

[18] X.-G. Zhao, G. A. Georgakis, and Q. Niu, Phys. Rev. B 56, 3976 (1997).

[19] Q. Sun and T. Lin, Phys. Rev. B 56, 3591 (1997).

[20] T. Martin and G. Berman, Phys. Lett. A 196, 65 (1994).

[21] C. A. Stafford and N. S. Wingreen, Phys. Rev. Lett. 76, 1916 (1996).

[22] S. Verghese, R. A. Wyss, Th. Schäpers, Q. Hu, A. Förster, and M. J. Rooks, Phys. Rev. B 52, 14834 (1995).

[23] T. H. Stoof and Yu. V. Nazarov, Phys. Rev. B 53, 1050 (1996).

[24] L. Y. Gorelik, F. A. Maaø, R. I. Shekhter, and M. Jonson, Phys. Rev. Lett. 78, 3169 (1997).

[25] G. B. Lesovik and L. S. Levitov, Phys. Rev. Lett. 72, 538 (1994).

[26] R. J. Schoelkopf, A. A. Kozhevnikov, D. E. Prober, and M. J. Rooks, Phys. Rev. Lett. 80, 2437 (1998).

[27] R. Landauer, Phil. Mag. 21, 863 (1970), M. Büttiker, Y. Imry, R. Landauer, and S. Pinhas, Phys. Rev. B 31, 6207 (1985); Y. Imry, Introduction to Mesoscopic Physics, Ox- 
ford University Press, New York, 1997.

[28] M. Büttiker, Phys. Rev. Lett. 57, 1761 (1986).

[29] M. Büttiker, A. Prêtre, and H. Thomas, Phys. Rev. Lett. 70, 4114 (1993).

[30] A. Prêtre, H. Thomas, and M. Büttiker, Phys. Rev. B 54, 8130 (1996).

[31] M. Büttiker, J. Phys.: Condens. Matter 5, 9361 (1993).

[32] Ya. M. Blanter and M. Büttiker, LANL preprint condmat/9706070; Y. M. Blanter, F. W. J. Hekking, and M. Büttiker, LANL preprint cond-mat/9710299.

[33] M. Büttiker and T. Christen in Mesoscopic Electron Transport, edited by L. L. Sohn, L. P. Kouwenhoven, and G. Schön, NATO ASI Series E: Applied Sciences, Vol. 345 (Kluwer, Dordrecht, 1997), p. 259.

[34] T. Christen and M. Büttiker, Phys. Rev. B 55, R1946 (1997).

[35] T. Christen and M. Büttiker, Europhys. Lett. 35, 523 (1996).

[36] An expansion beyond the quadratic order gives successively higher order conductances. See also J. Wang and H. Guo, Phys. Rev. B 54, R11090 (1996).

[37] W. Chen, T. P. Smith III, M. Büttiker, and M. Shayegan, Phys. Rev. Lett. 73, 146 (1994).
[38] J. S. Moon, J. A. Simmons, J. L. Reno, and B. L. Johnson, Phys. Rev. Lett. 79, 4457 (1997).

[39] M. Büttiker, Phys. Rev. B 46, 12485 (1992).

[40] T. Christen and M. Büttiker, Phys. Rev. Lett. 77, 143 (1996).

[41] P. W. Brouwer and M. Büttiker, Europhys. Lett. 37, 441446 (1997).

[42] Z-s. Ma, J. Wang, and H. Guo, LANL preprint condmat/9712060.

[43] M. H. Pedersen, S.A. van Langen, and M. Büttiker, Phys. Rev. B 57, 1838 (1998).

[44] M. Büttiker, J. Math. Phys. 37, 4793 (1996).

[45] M. Büttiker, H. Thomas, and A. Prêtre, Z. Phys. B 94, 133 (1994).

[46] M. Büttiker, IBM J. Res. Develop. 32, 63 (1988).

[47] G. Breit and E. Wigner, Phys. Rev. 49, 519 (1936).

[48] L. D. Landau and E. M. Lifshitz, Quantum Mechanics (Non-relativistic Theory). Pergamon Press, Oxford, 1977, p. 607.

[49] Y. Fu and S. C. Dudley, Phys. Rev. Lett. 70, 65 (1993).

[50] M. Holthaus, Phys. Rev. Lett. 69, 351 (1992); K. Drese and M. Holthaus, J. Phys.: Condens. Matter 8, 1193 (1996). 